\title{
Efficacy and Safety of Qingfei Paidu Decoction for Patients with COVID-19: A Systematic Review and Meta-Analysis
}

\author{
Zhen Yang ${ }^{1,2}$, Shuo Zhang ${ }^{3}$, Yu-Ping Tang ${ }^{1 \star}$, Sai Zhang ${ }^{1}$, Shi-Jun Yue ${ }^{1}$ and Qi-Ling Liu ${ }^{2 \star}$ \\ ${ }^{1}$ Key Laboratory of Shaanxi Administration of Traditional Chinese Medicine for TCM Compatibility, and State Key Laboratory \\ of Research \& Development of Characteristic Qin Medicine Resources (Cultivation), and Shaanxi Key Laboratory of Chinese \\ Medicine Fundamentals and New Drugs Research, and Shaanxi Collaborative Innovation Center of Chinese Medicinal Resources \\ Industrialization, Shaanxi University of Chinese Medicine, Xiłan 712046, Shaanxi Province, China \\ ${ }^{2}$ School of Public Health, Shaanxi University of Chinese Medicine, Xian 712046, Shaanxi Province, China \\ ${ }^{3}$ School of Clinical Medicine (Guanganmen Hospital), Beijing University of Chinese Medicine, Beijing 100029, China
}

\section{Asian Journal of Complementary and Alternative Medicine. Volume 10 Issue 01}

Published on: 24/01/2022

${ }^{*}$ Author for Correspondence: Yu-Ping Tang, Key Laboratory of Shaanxi Administration of Traditional Chinese Medicine for TCM Compatibility, and State Key Laboratory of Research \& Development of Characteristic Qin Medicine Resources (Cultivation), and Shaanxi Key Laboratory of Chinese Medicine Fundamentals and New Drugs Research, and Shaanxi Collaborative Innovation Center of Chinese Medicinal Resources Industrialization, Shaanxi University of Chinese Medicine, Xian 712046, Shaanxi Province, China, E-mail: yupingtang@sntcm.edu.cn

Qi-Ling Liu, School of Public Health, Shaanxi University of Chinese Medicine, Xi'an 712046, Shaanxi Province, China. E-mail: liuqilingsan@163.com.

Cite this article as: Yang Z, Zhang S, Tang YP, Zhang S, Yue SJ, Liu QL. Efficacy and Safety of Qingfei Paidu Decoction for Patients with COVID-19: A Systematic Review and Meta-Analysis. Asian Journal of Complementary and Alternative Medicine, Vol 10(1), 6-15:2022.

\begin{abstract}
Backgrounds: Since the outbreak of new coronavirus pneumonia worldwide, there are no specific drugs up to now. In people's struggle with the disease, more and more clinical practice shows that traditional Chinese medicine has shown an increasingly important role in the outbreak. Among them, Qingfei Paidu decoction (QFPD) has been widely used in China and overseas Chinese, which has good advantages in the treatment of COVID-19. Although some meta-analyses have studied the efficacy of QFPD in the treatment of COVID-19, the inclusion and exclusion criteria of trials in these studies were not strict enough and their results were not consistent. So, the exact efficacy of QFPD for treating COVID-19 was still questionable.
\end{abstract}

Objective: To systematically evaluate the efficacy and safety of QFPD for COVID-19.

Methods: A comprehensive literature search of qualified trials using QFPD to treat COVID-19 was conducted in 11 electronic databases, including Medline, Embase, Cochrane Library, PubMed, Web of Science, Springer link, Clinicaltrials.gov, etc. From their establishment to March 1, 2021. Subjects and abstracts of the trials were read in Note Express for preliminary screening, and the full text was read for further screening. Researchers independently extracted the data in duplicate, and the risk of bias of trials was assessed by using the Cochrane collaboration tool, followed by data analysis using Rev Man 5.3.

Results: Four trials with 390 participants were included for meta-analysis, revealing that QFPD can effectively shorten the average length of stay in hospital and the time of nucleic acid to turn negative. In terms of adverse reactions, there was no significant difference between the experimental and control groups.

Conclusions: These results demonstrated that QFPD could effectively treat COVID-19, improve clinical symptoms, shorten hospital stay and nucleic acid negative time, and no obvious adverse reactions have been found. Compared with other metaanalyses of QFPD, this research has some unique features, including its comprehensiveness, the large-scale of its search and its transparent approach, which may provide some new treatment ideas for clinicians.

Keywords: Qingfei Paidu decoction; COVID-19; 2019-NcoV; Systematic review; Meta-analysis 


\section{INTRODUCTION}

Corona Virus Disease 2019 (COVID-19) is an acute respiratory infectious disease caused by SARS-CoV-2, which is highly contagious. The novel coronavirus, as the seventh coronavirus that can infect humans discovered so far, belongs to the genus of coronavirus $\beta$, which is the same as MERS-CoV and SARS-CoV [1]. On February 11, 2020, the WHO named this new type of coronavirus pneumonia “COVID-19". Its clinical manifestations mainly include fever, dry cough, myalgia, fatigue, dyspnea, anorexia, diarrhea, ARDS, arrhythmia, acute kidney injury, different degrees of liver damage and septic shock [2]. As of March 5, 2021, the epidemic has caused 115658792 confirmed infections and 2572116 deaths worldwide. At present, the number of confirmed and death cases of COVID-19 is still increasing, which is straining worldwide healthcare capacity. Facing the battle between humans and viruses, many scholars at home and abroad continue to explore effective drugs for the treatment of COVID-19. Although early clinical trials have confirmed that various chemical drugs have a certain antiviral effect on SARS-CoV-2, the effectiveness of the drugs and security still needs further standardized research [3].

From "SARS" to "New Coronary Pneumonia", the participation of traditional Chinese medicine in major infectious diseases has shown its irreplaceable role [4]. Network pharmacology research also showed that in the prevention and treatment of COVID-19, Chinese medicine exerts antiviral, antiinflammatory and immunomodulatory effects through multiple components acting on multiple targets and multiple pathways [5]. QFPD was first seen in "Treatise on Febrile Diseases". It is a traditional Chinese medicine compound composed of Maxing Shigan Decoction, Shegan Mahuang Decoction, Xiao Chaihu, and Wuling Powder. It has been widely used in China to treat COVID-19. It contains 21 kinds of Chinese medicine ingredients, including Ephedrae Herba (Mahuang), Glycyrrhizae Radix Et Rhizoma Praeparata Cum Melle (Zhigancao), Armeniacae Semen Amarum (Xingren), Gypsum Fibrosum (Shengshigao), Cinnamomi Ramulus (Guizhi), Alismatis Rhizoma (Zexie), Polyporus (Zhuli), Atractylodis Macrocephalae Rhizoma (Baizhu), Poria(Fuling), Bupleuri Radix(Chaihu), Scutellariae Radix (Huangqin), Pinelliae Rhizoma (Banxia), Zingiberis Rhizoma Recens (Shengjiang), Asteris Radix Et Rhizoma (Ziyuan), Farfarae Flos (Donghua), Belamcandae Rhizoma (Shegan), Asari Radix Et Ehizoma(Xixin), Dioscoreae Rhizoma (Shanyao), Aurantii Fructus Immaturus (Zhishi), Citri Reticulatae Pericarpium (Chenpi), Agastacherugosus, Pogostemonis Herba (Guanghuoxiang) [6]. During the epidemic, the National Health Commission of the People's Republic of China formulated and issued the "New Coronavirus Pneumonia Diagnosis and Treatment Plan (Trial)" based on the clinical manifestations and pathological characteristics of the disease, with QFPD as the recommended prescription and included in the treatment plan [7]. However, there is still a lack of comprehensive and systematic evidence. Here we have conducted a systematic review and meta-analysis to evaluate the effect of QFPD on the efficacy and safety of COVID-19 patients. We hope that our research can provide the latest information for the treatment of COVID-19.

\section{DATA AND METHODS}

This study was conducted and reported in accordance with the Preferred Reporting Items for Systematic Reviews and Meta-analyses (PRISMA) [8] with Cochrane methodology. This study has been registered and the PROSPERO number is CRD42021242219.

\section{Literature search}

An electronic search of Cochrane Library, Embase, Medline, PubMed, Springer link, Web of Science, Clinicaltrials. gov, Chinese Biomedical Literature Database, the Chinese National Knowledge Infrastructure, Wanfang Database, VIP Database and Chinese Biomedical Literature Database was performed from the establishment of each electronic database to March 1, 2021. Forward and backward citation searching was conducted for all eligible trials. Following terms were used for searching: "COVID-19" OR "corona virus disease 2019" OR "coronavirus disease 2019" OR "severe acute respiratory syndrome coronavirus" OR "SARS-CoV-2" OR "novel corona virus" OR "novel coronavirus" OR “2019-nCoV” OR " $\mathrm{nCoV}$ 2019”) AND ("qingfeipaidu” OR "Qingfei Paidu decoction" OR "qing fei pai du") AND ("clinical trial" OR "randomized controlled trial" OR "randomized controlled trial" OR "lin chuang yan jiu" OR "lin chuang shi yan"). The language and status of publications in our literature search were not specified.

\section{Information sources and eligibility criteria}

Types of trials: This study included randomized controlled trials or retrospective trials in which QFPD were used to treat COVID-19. Trials were excluded if: (a) no control group was used; (b) QFPD was not used in the experimental group; (c) combined with other drugs; (d) trials on effective analysis data cannot be obtained; (e) reviews, conference paper, case reports, experience sharing, animal trials, etc.; (f) repeatedly published articles and plagiarized trials.

Types of participants: Patients who were not restricted by age, gender, or nationality with COVID-19 were eligible for inclusion in this study. The new coronary pneumonia standard 
refers to the "New Coronavirus Pneumonia Diagnosis and Treatment Program".

\section{Types of interventions}

The intervention measures in the experimental group should contain QFPD, and the control group should be chemical drugs (CD).

\section{Types of outcome measures}

The main indicators included the time for nucleic acid to become negative and the length of hospital stay, and the secondary indicator is adverse reactions.

\section{Study selection and data extraction}

According to the inclusion and exclusion criteria mentioned above, two researchers who participated in calibration and training exercises before starting the screening processes independently screened the titles and abstracts of potential eligible trials which were in duplicate, then they retrieved independently and reviewed the full text of the possible trials in duplicate based on the inclusion and exclusion criteria and compared their results. The screening process was conducted in Note Express 3.2.0.

We conducted various forms of calibration exercises and pilots before the data extraction process began. Two researchers used standardized tables to independently extract data in duplicate from all eligible trials according to the inclusion and exclusion criteria mentioned above. In case of disagreement, they agreed through discussion or submitted it to a third party for evaluation. And before the screening process, the third party used a standardized screening form and performed calibration exercises.

For all eligible trials, the researchers extracted data on the following characteristics: the basic information of the study (author's name, title of the study, year of publication, country/ region, publication status), study characteristics (sample size, source of cases, age, diagnostic criteria, inclusion and exclusion criteria), intervention and control measures (dosage form, dose and duration), research methodology (random scheme generation, allocation hiding, blind method, incomplete result data, selective reporting, other biases, loss of follow-up) and outcome measures.

\section{Quality assessment}

The methodological quality of each included study was assessed independently by two reviewers according to 2 tools. The Cochrane collaboration tool has been used to assess the quality of randomized controlled trials. It comprised the following 7 aspects: random sequence generation, allocation concealment, blind method, incomplete result data, selective reporting, and other biases. The quality assessment results of each item can be divided into three grades: "low risk", "high risk" and "unclear". The more rigorous the design and the higher the methodological quality of each RCT, the lower the risk coefficient. Newcastle Ottawa Scale (NOS) has been used to assess the quality of retrospective studies. This method includes 3 aspects of evaluation: the selection method, comparability and contact exposure assessment method of case group and control group. The higher the score, the higher the quality of the study. When necessary, the consensus on this issue was studied with the help of a third party.

\section{Data analysis}

Data analysis was performed using Rev Man 5.3 software. Both the continuous and dichotomous outcomes were derived from the included trials without any conversion. The dichotomous outcomes were described by relative risk (RR) and $95 \%$ confidence interval (CI), in addition, mean difference (MD) and 95\% CI were used to describe the effect value of the continuous comparison. Heterogeneity was determined according to the results of $\mathrm{I}^{2}$ test. $\mathrm{I}^{2}<50 \%$ indicated the low heterogeneity of inter-study, and the fixed effect model was adopted. Furthermore, the random effect model was adopted when $\mathrm{I}^{2}>50 \%$. A random effect model was also used to generate direct and mixed treatment comparison estimates. Subgroup analysis was conducted according to whether the experimental group was combined with $\mathrm{CD}$ and the different treatment methods in the control group.

\section{RESULTS}

\section{Study identification}

Based on the above retrieval strategy, a total of 1065 potentially relevant trials were retrieved from 11 electronic databases, and 235 trials were retrieved after duplicates were deleted. After reviewing the titles and abstracts, 222 trials were excluded because they did not comply with the inclusion criteria, and 13 trials initially met the predetermined requirements and their full texts were read for detailed assessment. Finally, 4 trials [9-12] were included for meta-analysis. The PRISMA flow diagram of the literature retrieval process was shown in Figure 1. All included trials have been published as a full article.

\section{Study characteristics}

Table 1 summarized the basic characteristics of the eligible 4 trials, all of which were conducted in China. A total of 390 patients with COVID-19 were analyzed. Sample sizes ranged from 12 to 229 . Among them, 2 trials $[9,10]$ were randomized controlled trials, $2[11,12]$ were retrospective studies. QFPD combined with CD vs. CD was used in these included trials. 

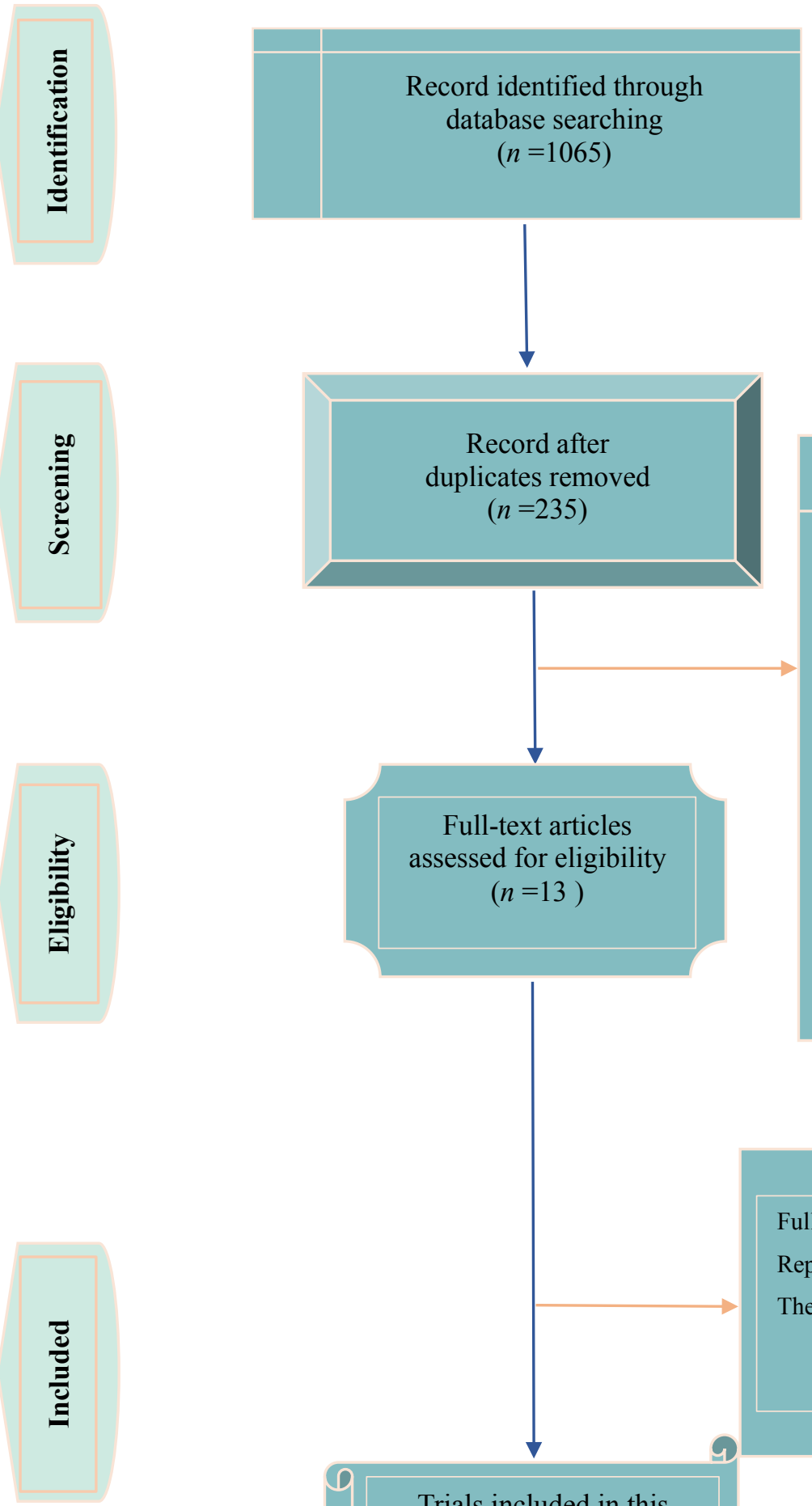

Title and abstract excluded, with reasons

$$
(n=222)
$$

Review(Including Meta-analysis $) \quad(n=127)$

Medicine research $(n=62)$

Case report $(n=11)$

Animal experiment $\quad(n=8)$

Descriptive research $(n=3)$

The grouping is not required. $(n=11)$

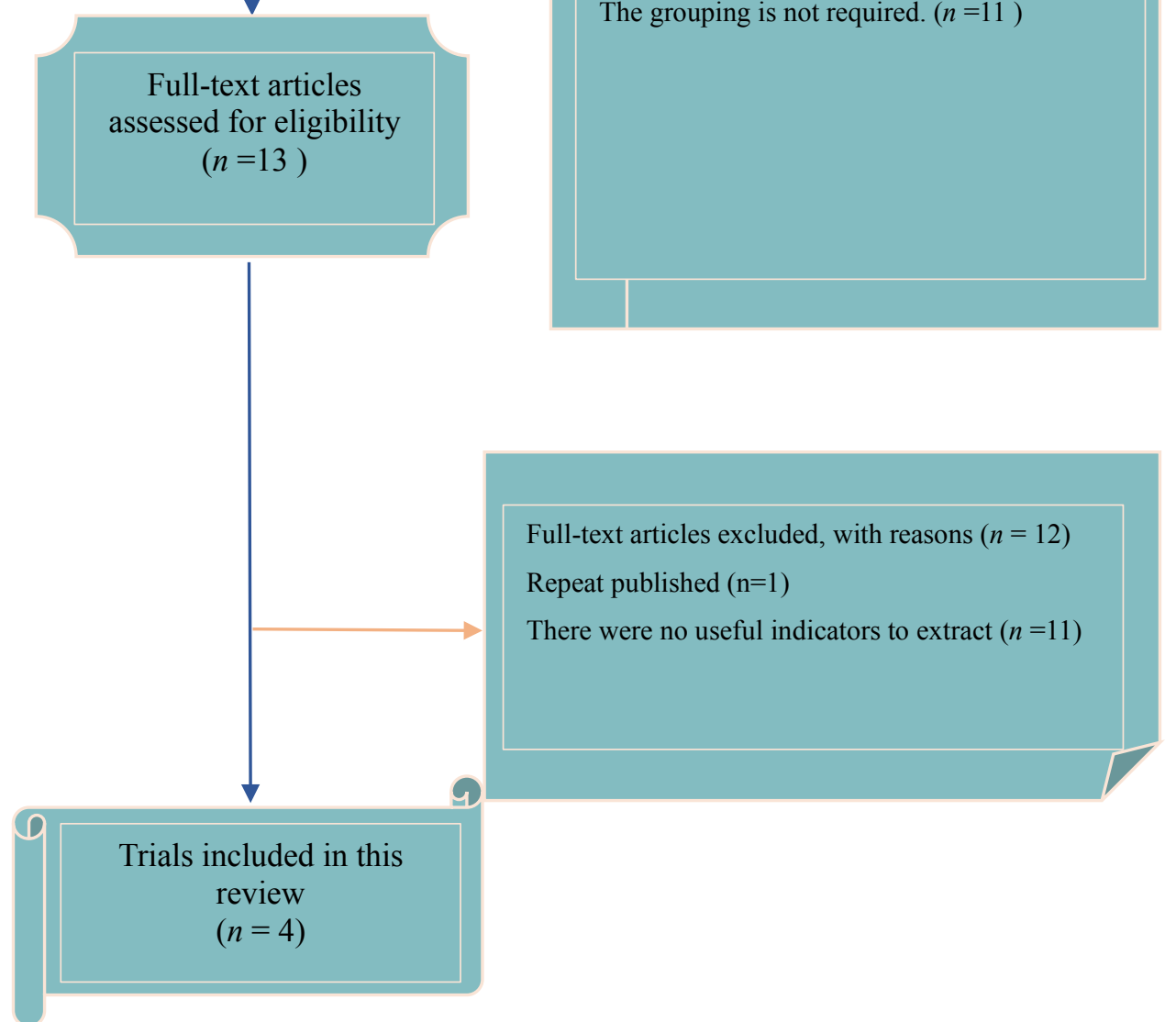

Figure 1: PRISMA flow diagram of literature selection. 
Table 1: Characteristic of the 4 trials included in the meta-analysis.

\begin{tabular}{|c|c|c|c|c|c|}
\hline Author(s) & $\begin{array}{l}\text { Sample size } \\
\text { (experimental/ } \\
\text { control) }\end{array}$ & $\begin{array}{l}\text { Patient enrollment } \\
\text { time }\end{array}$ & Experimental & Control & Outcome measures \\
\hline Zeng et al, 2020 & $104 / 125$ & 2019.12-2020.3 & QFPD+CD & $\mathrm{CD}$ & $\begin{array}{l}\text { SAS SDS score, Comparison of incidence of } \\
\text { complications (pneumonia, acute respiratory } \\
\text { disease, shock, total incidence), Comparison } \\
\text { of clinical efficacy (chest image improvement } \\
\text { rate, nucleic acid negative conversion, discharge } \\
\text { time) }\end{array}$ \\
\hline Yu et al, 2020 & $43 / 46$ & 2020.2.10-2020.4.10 & $\mathrm{QFPD}+\mathrm{CD}$ & $\mathrm{CD}$ & $\begin{array}{l}\text { Blood routine and inflammatory factors, } \\
\text { Cellular immunity level, The biochemical } \\
\text { indicators, Blood coagulation function, } \\
\text { Indicator value difference comparison,Chest } \\
\text { computed tomography }(\mathrm{CT}) \text {, The length of } \\
\text { hospital stay and the time of nucleic acid } \\
\text { turning negative }\end{array}$ \\
\hline Li et al, 2020 & $60 / 60$ & 2020.1.24-2020.3.7 & $\mathrm{QFPD}+\mathrm{CD}$ & $\mathrm{CD}$ & $\begin{array}{l}\text { Clinical effective rate, Symptoms (fever, cough, } \\
\text { exacerbation) and lung CT, Adverse reactions }\end{array}$ \\
\hline Li and Zhang, 2020 & $6 / 6$ & $2020.2-2020.3$ & QFPD+CD & $\mathrm{CD}$ & $\begin{array}{l}\text { Treatment status (WBC count, } \mathrm{PCO}, \mathrm{PO} 2, \\
\text { length of hospital stay,), Clinical curative effect, } \\
\text { Adverse reactions }\end{array}$ \\
\hline
\end{tabular}

Abbreviations: $\mathrm{CD}$ : Chemical drugs

1. SAS SDS score

2. Comparison of incidence of complications (pneumonia, acute respiratory disease, shock, total incidence)

3. Comparison of clinical efficacy (chest image improvement rate, nucleic acid negative conversion, discharge time)

4. Blood routine and inflammatory factors

5. Cellular immunity level

6. The biochemical indicators

7. Blood coagulation function

8. Indicator value difference comparison

9. Chest computed tomography (CT)

10. The length of hospital stay and the time of nucleic acid turning negative

11. Clinical effective rate

12. Symptoms (fever, cough, exacerbation) and lung CT

13. Adverse reactions

14. Treatment status (WBC count, $\mathrm{PCO} 2, \mathrm{PO} 2$, length of hospital stay,)

15. Clinical curative effect

16. Adverse reactions

\section{Quality of including trials}

The methodological quality of 2 randomized controlled trials $[9,10]$ was summarized in Figure 2 and the criteria in the Cochrane Handbook for Systematic Reviews of Interventions were used to assess the risk of bias in the study. Randomization was announced in only one of the 4 trials [10], and all of the trials did not report allocation concealment and blind method. The quality of 2 retrospective studies $[11,12]$ was assessed by NOS. Table 2 summarized the NOS scores of each study. All the studies were of fair quality. Figure 3 provided the forest plot of the trials, which showed hospitalization time in different interventions. Figure 4 provided the forest plot of the trials, which showed an adverse reaction in different interventions. Figure 5 provided the forest plot of the trials, which showed nucleic acid conversion time in different interventions. 


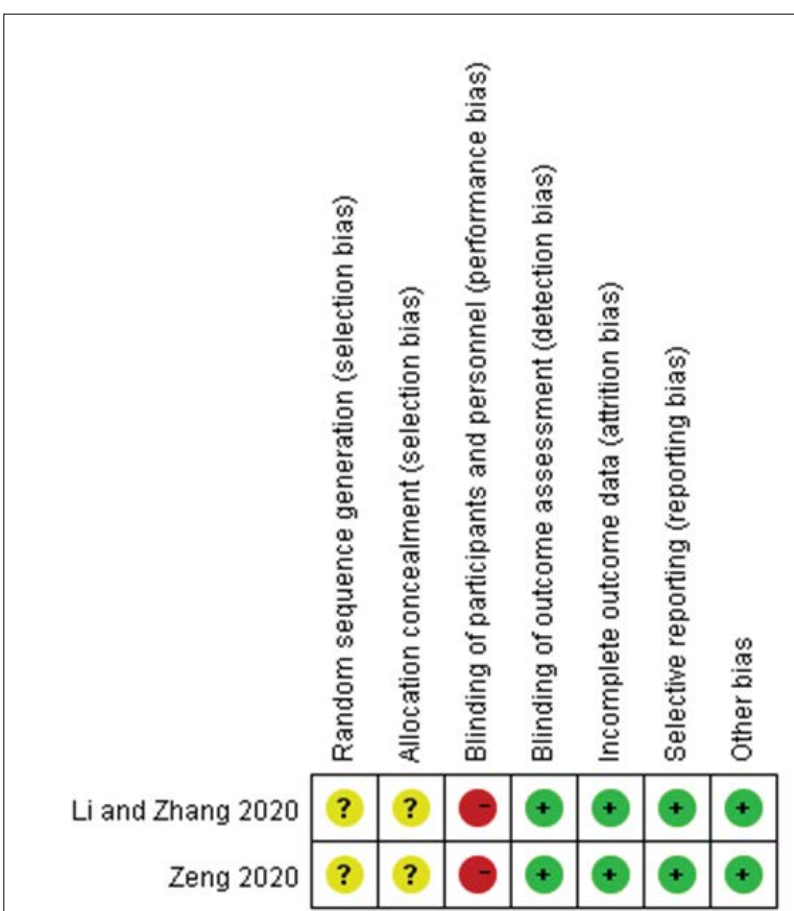

Figure 2: Methodological quality assessment results for all included trials.
Table 2: Results of quality assessment for retrospective studies by using NOS scale.

\begin{tabular}{|l|c|c|}
\hline \multirow{2}{*}{ Items } & \multicolumn{2}{|c|}{$\begin{array}{c}\text { References } \\
\text { (scores) }\end{array}$} \\
\cline { 2 - 3 } & $\begin{array}{c}\text { Yu et } \\
\text { al., 2020 }\end{array}$ & $\begin{array}{c}\text { Li et al., } \\
\mathbf{2 0 2 0}\end{array}$ \\
\hline The selection of participants (4 scores) & & \\
\hline Representativeness of the exposure group & 1 & 1 \\
\hline Selection of non-exposed groups & 1 & 1 \\
\hline Determination of exposure factors & 1 & 1 \\
\hline $\begin{array}{l}\text { There were no outcome indicators to } \\
\text { look for at the start of the study }\end{array}$ & 0 & 0 \\
\hline Intergroup comparability (2 scores) & & \\
\hline $\begin{array}{l}\text { Comparability between exposed and } \\
\text { non-exposed groups was considered in } \\
\text { the design and statistical analysis }\end{array}$ & 2 & 2 \\
\hline Outcome measurement (3 scores) & & \\
\hline Evaluation of outcomes & 1 & 1 \\
\hline Follow-up was long enough & 0 & 0 \\
\hline $\begin{array}{l}\text { Complete follow-up of the exposure } \\
\text { group and the exposure group }\end{array}$ & 0 & 0 \\
\hline Total & 6 & 6 \\
\hline
\end{tabular}

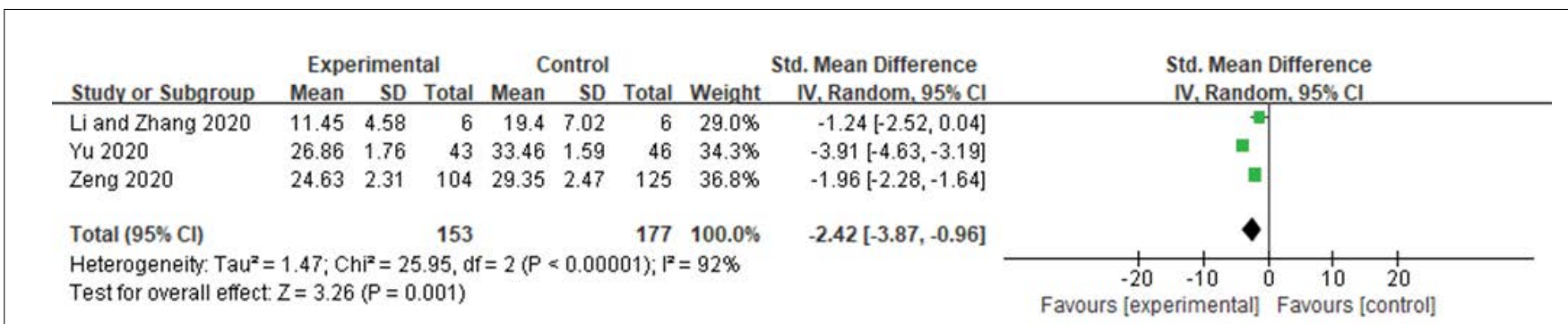

Figure 3: Forest plot of the trials showing hospitalization time in different interventions.

\begin{tabular}{|c|c|c|c|c|c|c|c|c|c|c|c|}
\hline \multirow{3}{*}{$\begin{array}{l}\text { Study or Subgroup } \\
\text { Yu } 2020 \\
\text { Zeng } 2020\end{array}$} & \multicolumn{3}{|c|}{ Experimental } & \multicolumn{3}{|c|}{ Control } & Weight & $\begin{array}{l}\text { Mean Difference } \\
\text { IV. Random, } 95 \% \mathrm{Cl}\end{array}$ & \multicolumn{3}{|c|}{$\begin{array}{c}\text { Mean Difference } \\
\text { IV. Random, } 95 \% \mathrm{Cl}\end{array}$} \\
\hline & 23.37 & 1.6 & 43 & 27.61 & 1.61 & 46 & $47.8 \%$ & $-4.24[-4.91,-3.57]$ & 들 & & \\
\hline & 5.36 & 1.25 & 104 & 10.63 & 2.62 & 125 & $52.2 \%$ & $-5.27[-5.79,-4.75]$ & $\mathbf{\square}$ & & \\
\hline Total $(95 \% \mathrm{Cl})$ & & & 147 & & & 171 & $100.0 \%$ & $-4.78[-5.79,-3.77]$ & $\bullet$ & & \\
\hline $\begin{array}{l}\text { Heterogeneity: Tauz } \\
\text { Test for overall effec }\end{array}$ & $\begin{array}{l}0.44 ; C r \\
Z=9.29\end{array}$ & $\begin{array}{l}h i^{2}=5 . \\
(P<0\end{array}$ & $\begin{array}{l}71, \mathrm{df}= \\
.00001)\end{array}$ & $1(P=$ & $0.02) ;$ & $I^{2}=82 \%$ & & & $\begin{array}{cc}1 & 1 \\
-20 & -10 \\
\text { Favours [experimental] }\end{array}$ & $\begin{array}{l}1 \\
0 \\
\text { Favours [control] }\end{array}$ & 20 \\
\hline
\end{tabular}

\section{Outcomes}

This study involved 3 outcome indicators, including length of stay in the hospital, time to negative nucleic acid, and adverse reactions. A total of 3 trials [9-11] reported the length of stay in the hospital, 2 trials $[9,11]$ reported the time for nucleic acid to become negative, and 2 trials $[10,12]$ reported the adverse reactions. Meta-analysis showed that using QFPD treat COVID-19 could effectively shorten the length of hospital stay ( 3 trials, $n=330$, MD: $-2.42 ; 95 \% \mathrm{CI}:-3.87$ to $-0.96 ; p=0.001$; Figure 3 ) and shorten the time of nucleic acid conversion ( 2 trials, $n=318, \mathrm{MD}:-4.78$; 95\% CI: -5.79 to $-3.77 ; p=0.02$; Figure 4 ). Additionally, no obvious adverse 


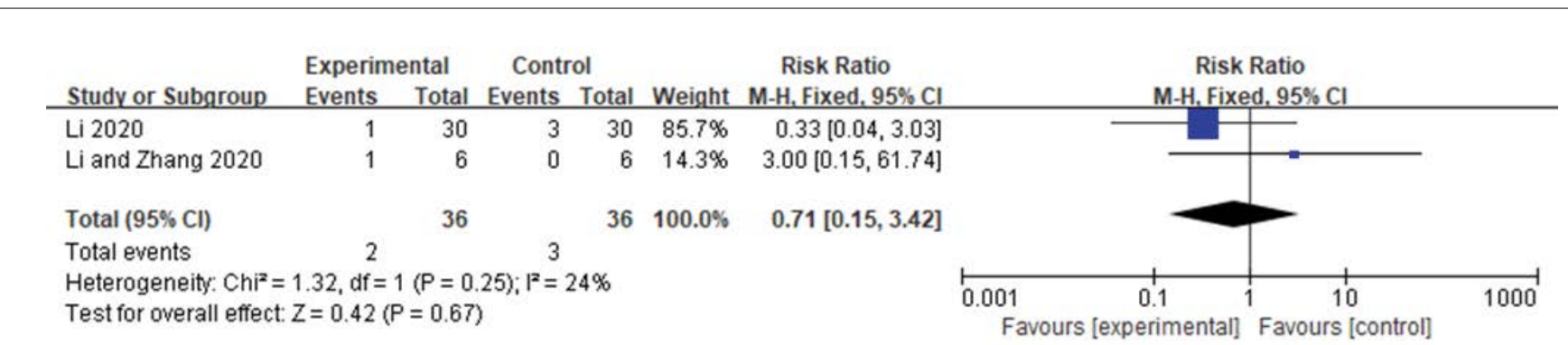

Figure 5: Forest plot of the trials showing adverse reaction in different interventions.

reactions were found between the experimental group and the control group ( 2 trails, $n=72$; RR: $0.71 ; 95 \%$ CI: 0.15 to 3.42; $p<0.00001$; Figure 5).

\section{DISCUSSION}

\section{Summary of evidence}

The efficacy and safety of QFPD for COVID-19 were evaluated by meta-analysis based on 4 trials [9-12] and 390 participants. The results showed that compared with the use of $\mathrm{CD}$ alone, QFPD combined with CD can be effective in disease control to a certain extent. It was manifested in shortening the length of hospitalization and nucleic acid transfer time. Additionally, no obvious adverse reactions have been found.

Although there have been some meta-analysis studies on the clinical efficacy of QFPD in the treatment of COVID-19, they have certain limitations. Some data retrieval scope was small, resulting in insufficient comprehensive results; some inclusion and exclusion standards were not strict enough, leading to biased results; some research designs were singlecenter, and the results were not representative enough and some do not have specific data processing. Therefore, these studies cannot reflect the efficacy of QFPD in the treatment of COVID-19 well. This study has some special features, including comprehensive and large-scale searches, standardized and strict inclusion and exclusion criteria, and transparent methods. And it is hoped that it will provide new support for the treatment of COVID-19. Based on this, we hope that our research can provide new ideas for the treatment of COVID-19.

QFPD plays an anti-inflammatory, anti-viral, and immune role in the treatment of COVID-19 through the comprehensive effects of multiple drugs. The main mechanism of Ephedrae Herba is alkaloids and ESP, Alkaloids play an anti-asthma effect by promoting the release of neurotransmitters such as norepinephrine and epinephrine. In addition, it can directly stimulate $\beta$-adrenergic receptors and $\alpha$-adrenergic receptors. Energy receptors relax and contract bronchial smooth muscles [13]. Glycyrrhizae Radix Et Rhizoma Praeparata Cum Melle is a plant with the same medicine and food, and its main ingredient glycyrrhizic acid has anti-inflammatory and anti-cancer effects [14]. Cinnamomi Ramulus contains volatile oils such as cinnamyl alcohol and cinnamaldehyde, as well as organic acids, mainly cinnamic acid. It has antipyretic, analgesic, antibacterial, antiviral, vasodilator, antioxidant, and diuretic effects [15]. Gypsum Fibrosum has heat-clearing, analgesic and anti-inflammatory effects. Bitter almonds are rich in chemical components, more active substances, and high in amygdalin, which can be decomposed into hydrocyanic acid and benzoic acid in the body. Hydrocyanic acid can inhibit the respiratory center and achieve the effect of antitussive and antiasthmatic [16]. The rhizome of Atractylodis Macrocephalae Rhizoma has a variety of biological activities, including improving gastrointestinal function, as well as anti-tumor, anti-inflammatory, anti-aging, anti-oxidant, antiosteoporosis, antibacterial and neuroprotective activities [16]. Poria and some of its active ingredients have significant antibacterial and anti-tumor effects, with low toxic and side effects, and also play an important role in cancer treatment [17]. The saikosaponins in Bupleuri Radix can effectively inhibit inflammatory exudation, promote the release of inflammatory mediators, increase migrating white blood cells, proliferate connective tissue, and inhibit the occurrence of allergic inflammation [18]. Studies have reported that Scutellariae Radix extracts and isolated compounds have a variety of pharmacological properties, including antitumor, anti-inflammatory, neuroprotective, anti-mutation, anti-convulsive, and anti-oxidant properties [19]. Zingiberis Rhizoma Recens can achieve anticancer effects through factors such as free radical scavenging, antioxidant pathways, gene expression changes and apoptosis induction, while ginger can effectively relieve symptoms such as dyspnea [20]. The volatile oil of Asari Radix Et Ehizoma is considered to be an effective component molecule for anti-inflammatory pain [21], which can effectively relieve respiratory symptoms; Citri Reticulatae Pericarpium has a wide range of pharmacological 
effects, among which flavonoids are closely related to the pharmacological activity of Citri Reticulatae Pericarpium, and hesperidin is its main active ingredient One of them has various activities such as regulating blood lipids, antiinflammatory, and anti-oxidant [22]. Pogostemonis Herba has always been used in traditional Chinese medicine to treat inflammatory diseases. Its main active ingredient, PA, has a good anti-inflammatory effect [23]. The main ingredients in Belamcandae Rhizoma are flavonoids, triterpenoids, quinones and phenolic compounds, which have antiinflammatory and antibacterial properties. Anti-virus, elimination of free radicals, anti-allergic and other biological activities and pharmacological effects [24]. Clinically, it is mainly used to treat bronchial asthma, bronchitis, chronic obstructive pulmonary disease and other pulmonary diseases. Dioscin can reduce OVA-induced allergic airway inflammation by inhibiting NF- $\mathrm{BB}$ activation [25]. Three main types of chemical components are included in Aurantii Fructus Immaturus. Among them, flavonoids account for a relatively high proportion (5\% to $28 \%$ of the total) [26] and their main functions include regulating gastrointestinal function, anti-oxidation and anti-tumor activity, etc.; volatile oils are the main components that produce Qi regulation and have an antitussive antibacterial effect [27] and alkaloids have the functions of dispelling wind and regulating qi, warming the stomach, promoting appetite, and promoting metabolism [28]. Additionally, flavonoids and polysaccharides also have good antioxidant activity [28]. Organic acids are the main chemical components of Pinelliae Rhizoma, which can effectively relieve cough and expectorant. The alkaloids, water-soluble organic acids, protein and polysaccharides contained in Pinelliae Rhizoma all have antiemetic activity [29].

Traditional Chinese medicine has rich experience in conditioning the body and enhancing resistance to epidemics. For mild and ordinary patients, early intervention of Chinese medicine can effectively prevent the disease from turning into a critical condition [30]. Basic research has found that QFPD has anti-inflammatory, anti-viral and immune regulation effects. Chen's research also showed that QFPD could resist COVID-19 virus infection by regulating metabolism and pro-inflammatory processes [31]. This showed that QFPD is suitable for mild and ordinary patients and effective for severe patients. In the future, more research is still needed to verify its exact efficacy, and tap more potential effects, which can make QFPD plays a better role in public health.

\section{LIMITATIONS}

The limitations of this study should be noted. Firstly, because most of the current clinical trials on QFPD in the treatment of COVID-19 were retrospective studies and only a few were randomized controlled trials, which with insufficient sample size and short duration, the methodological quality was subject to certain risk bias. Secondly, as the application of TCM in other countries is limited, QFPD is mainly used in China. Although the Chinese government has issued health packages (included QFPD) to Chinese people worldwide, the data cannot be calculated and summarized well. Thus, a strict randomized well-controlled trial could hardly be achieved. Owing to the limited sample size, long-term randomized controlled trials with follow-up evaluations are still required to confirm the present results.

\section{CONCLUSIONS}

In summary, this study found that QFPD may be an effective drug for the treatment of COVID-19. Combining with chemical drugs can significantly improve clinical symptoms and demonstrate better clinical effects, including inhibiting the development of the disease, shortening the length of hospitalization and the time for nucleic acid to become negative. Also, no obvious adverse reactions were found. Owing to the limited sample size, long-term randomized controlled trials with follow-up evaluations are required to confirm the results presented here.

\section{DATA AVAILABILITY}

The data used to support the findings of this study are available from the corresponding author upon request.

\section{CONFLICTS OF INTEREST}

The authors declare that they have no conflicts of interest.

\section{AUTHORS' CONTRIBUTIONS}

Zhen Yang, Yu-Ping Tang and Qi-Ling Liu were responsible for the conception and design of the study; Zhen Yang, Shuo Zhang, Sai Zhang and Shi-Jun Yue conducted the statistical analysis, drew the tables and pictures, and drafted the manuscript; Zhen Yang and Shuo Zhang retrieved the database, screened the trials, extracted the data, and evaluated the methodological quality; and all authors critically revised the manuscript and approved the final version.

\section{ACKNOWLEDGEMENTS}

This study was funded by the National Natural Science Foundation of China (81773882, 81974584), and Key Research and Development Program of Shaanxi Province (2019ZDLSF04-05, 2019SF-295). This research was also financially supported by Subject Innovation Team of Shaanxi University of Chinese Medicine (2019-YL10). 


\section{REFERENCES}

1. Liya G, Yuguang W, Jian L, Huaiping Y, Xue H, et al. (2020) Studies on viral pneumonia related to novel coronavirus SARSCoV-2, SARS-CoV, and MERS-CoV: A literature review. APMIS 128: 423-432.

2. Wang Y, Wang Y, Chen Y, Qin Q (2020) Unique epidemiological and clinical features of the emerging 2019 novel coronavirus pneumonia (COVID-19) implicate special control measures. J Med Virol 92: 568-576.

3. Pan X, Dong L, Yang L, Chen D, Peng C (2020) Potential drugs for the treatment of the novel coronavirus pneumonia (COVID-19) in China. Virus Res 286: 198057.

4. Zhang CX (2020) Constructing a new public health governance model with Chinese Medicine. China Health Law 28: 80-86.

5. Dai YJ, Wan SY, Gong SS, Liu JC, Li F, et al. (2020) Recent advances of traditional Chinese medicine on the prevention and treatment of COVID-19. Chin J Nat Med 18: 881-889.

6. Chen J, Wang YK, Gao Y, Hu LS, Yang JW, et al. (2020) Protection against COVID-19 injury by qingfei paidu decoction via antiviral, anti-inflammatory activity and metabolic programming. Biomed Pharmacother 129: 110281.

7. Gao K, Song YP, Chen H, Zhao LT, Ma L (2020) Therapeutic efficacy of Qingfei Paidu decoction combined with antiviral drugs in the treatment of corona virus disease 2019: A protocol for systematic review and meta analysis. Medicine (Baltimore) 99: e20489.

8. Moher D, Liberati A, Tetzlaff J, Altman DG, The PRISMA Group (2009) Preferred reporting items for systematic reviews and meta-analyses: The PRISMA statement. PLoS Med 6: e1000097.

9. Zeng XH, Ma WH, Wang J (2020) Effect of Qingfei Paidu decoction on clinical efficacy of COVID-19 pneumonia with Phlegm heat blocking lung. Med J West Chin 32: 1799-1806.

10. Li Y, Zhang W (2020) Evaluation on the Clinical Effect of Traditional Chinese Medicine and Western Medicine Regimens on COVID-19. Guangming Journal of Chinese Medicine 35: 1273-1275.

11. Yu XY, Zhang S, Yan FF, Su DZ (2020) Comparison of clinical efficacy of Qingfei Paidu decoction combined with western medicine in 43 cases and single western medicine in 46 cases in the treatment of COVID-19. J Shandong Univ (Health Sciences) 58: 47-53.

12. Li KY, An W, Xia F, Chen M, Yang P, et al. (2020) Observation on clinical effect of modified Qingfei Paidu Decoction in treatment of COVID-19. Chin Tradit Herbal Drugs 51: 2046-2049.

13. Miao SM, Zhang Q, Bi XB, Cui JL, Wang ML (2020) A review of the phytochemistry and pharmacological activities of Ephedra herb. Chin J Nat Med 18: 321-344.

14. Chen M, Zhu J, Kang J, Lai X, Gao Y, et al. (2019) Exploration in the Mechanism of Action of Licorice by Network Pharmacology. Molecules 24: 2959.

15. Wang HW, Wu ZB, Yang M, Liu X, Lv BJ, et al. (2020) Research Overview of Modern Pharmacological Effects of Guizhi Decoction. Jiangsu Journal of Traditional Chinese Medicine 52: 85-89.

16. Zhu B, Zhang QL, Hua JW, Cheng WL, Qin LP (2018) The traditional uses, phytochemistry, and pharmacology of Atractylodes macrocephala Koidz: A review. J Ethnopharmacol 226: 143-167.

17. Wang N, Liu D, Guo J, Sun Y, Guo T, et al. (2018) Molecular mechanism of Poria cocos combined with oxaliplatin on the inhibition of epithelial-mesenchymal transition in gastric cancer cells. Biomed Pharmacother 102: 865-873.

18. Yang F, Dong X, Yin X, Wang W, You L, et al. (2017) Radix Bupleuri: A Review of Traditional Uses, Botany, Phytochemistry, Pharmacology, and Toxicology. Biomed Res Int 2017: 21-22.

19. Sowndhararajan K, Deepa P, Kim M, Park SJ, Kim S (2018) Neuroprotective and Cognitive Enhancement Potentials of Baicalin: A Review. Brain Sci 8: 104.

20. Townsend EA, Siviski ME, Zhang Y, Xu C, Hoonjan B, et al. (2013) Effects of ginger and its constituents on airway smooth muscle relaxation and calcium regulation. Am J Respir Cell Mol Biol 48: 157-163.

21. Jing Y, Zhang YF, Shang MY, Liu GX, Li YL, et al. (2017) Chemical Constituents from the Roots and Rhizomes of Asarum heterotropoides var. mandshuricum and the In Vitro Anti-Inflammatory Activity. Molecules 22: 125.

22. Yu X, Sun S, Guo Y, Liu Y, Yang D, et al. (2018) Citri Reticulatae Pericarpium (Chenpi): Botany, ethnopharmacology, phytochemistry, and pharmacology of a frequently used traditional Chinese medicine. J Ethnopharmacol 220: 265-282.

23. Zhang W, Zhang JJ, Guo QF, Chen L, Yi ZH (2020) Advances in Pharmacological Studies of Patchouli Alcohol. Chinese Journal of Experimental Traditional Medical Formulae 26: 213-221.

24. Zhou XH (2014) Pharmacological analysis of the treatment of chronic pharyngitis by atomization of Radix Artemisiae, Honeysuckle and Licorice. Guangming Journal of Chinese Medicine 29: 855-856.

25. Wu LJ, Wei HL, Tong YN (2021) Dioscin alleviates airway inflammation in ovalbumin-induced allergic asthma mice. Immunological Journal 37: 210- 216.

26. Huang XL, Chen YY (2016) Study on the Contents of Effective Components in Fructus Aurantii Immaturus and Its Processed Products by HPLC. Guangzhou Chemical Industry 44: 22-49.

27. Lv QL, Feng F (2013) Comparative Study on Main Chemical Constituents of Citrus aurantii and Citrus aurantia. Journal of North Pharmacy 10: 8-9. 
28. Zhang XJ, Zhao LY, Li JH, Zhang N, Fan ZW (2021) Research Review on Fructus aurantii Immaturus. Acta Chinese Medicine and Pharmacology 49: 94-100.

29. Wang YM, Wang QH (2020) Research progress on chemical constituents, pharmacological effects and toxicity of Pinellia ternate. China Pharmacy 31: 2676-2682.
30. Ren JL, Zhang AH, Wang XJ (2020) Traditional Chinese medicine for COVID-19 treatment. Pharmacol Res 155: 104743.

31. Chen J, Wang YK, Gao Y, Hu LS, Yang JW, et al. (2020) Protection against COVID-19 injury by qingfei paidu decoction via antiviral, anti-inflammatory activity and metabolic programming. Biomed Pharmacother 129: 110281. 